\title{
DLBCL of Clavicle: Role of FNA in Diagnosis of Rare Lesion
}

\author{
Cyrus Dara Jokhi, Kalpesh V. Vaghela and Pruthvi Damor
}

Department of Pathology, Zydus Medical College and Hospital, Dahod. (389151) State - Gujarat Country - India

\section{ABSTRACT}

Primary bone lymphoma israre, among which commonly involved site at time of presentationare femur, pelvic bones, tibia and most common type is Large B cell lymphoma which usually occurs in adults. Cases of large B cell lymphoma in clavicle as primary bone lymphoma is extremely rare finding. According to our knowledge this case may be first reported case of world. We are here reporting a case of 62-year female presented as sternoclavicular joint swelling, without fever or pain in local area. Patient was diagnosed on X ray by radiologist, as well as by clinician as, osteosarcoma of right clavicle. Patient came to Department of Cytopathology for FNA for confirmation of X ray finding. On FNA diagnosis of Non-Hodgkin lymphoma was given, and advise for biopsy and immunohistochemistry(IHC) was given for confirmation of diagnosis and to subtype NHL. On IHC Ki67 index was 85\%, with diffuse positivity noted for MUM1, CD79a, and CD138 along with cytoplasmic positivity for ALK 1 and patchy positivity for PAX 5. Tumor was negative for BCL6, BCL2, CD20, CD5, CD23, CD10, CD19, CD4, CD8, CD7, CD3. Diagnosis of ALK positive DLBCL was given on biopsy after IHC. Patients with PBL treated with combined modality were found to have a superior outcome, with a significantly better survival (5-year cause-specific survival 95\%). So correct and timely pathological diagnosis of DLBCL as PBL is significantly important for prognosis.

Keywords: Primary Bone Lymphoma, Osteosarcoma, Clavicle.

\section{Introduction}

World Health Organization defines the criteria for a diagnosis of PBL as (1) a single skeletal tumor without regional lymph node involvement, and (2) multiple bone lesions without visceral or lymph node involvement.

Though secondary involvement of the bone marrow is a common event in systemic lymphomas, but Primary bone lymphomas are rare. Except Diffuse large B-cell lymphomas other types of primary bone lymphomas with a rare occurrence; are Follicular, Marginal zone, Anaplastic large cell, Hodgkin, and T-cell lymphomas. The Primary bone lymphomas affects the middle-aged to elderly population. The patients usually present with local symptoms like bone pain, palpable mass or fractures, systemic symptoms, such as fever, night sweats and weight loss, occur less frequently in PBL when compared with other NHL. The metaphysis of bones is a common location of Primary bone lymphomas. ${ }^{(2)}$ Primary bone lymphoma is rare, among which commonly involved site at time of presentation are femur, pelvic bones, tibia. ${ }^{[1]}$ Cases of large $\mathrm{B}$ cell lymphoma in clavicle as primary bone lymphoma is extremely rare finding. The prognosis of $\mathrm{PBL}$ is relatively good and equal to that of samestage systemic DLBCL. Based on the experience of the diagnosis of present case, diagnosis of PBL only by using radio imaging is difficult, since the findings are not pathognomonic.
Histopathological examination may define the diagnosis and avoid the delay in treatment. Previous studies have reported the use of radiotherapy, chemotherapy or a combination of the two for the treatment of PBL, resulting in a generally good prognosis. The radiotherapy dose ranges from 2000-6150 centi-gray (cGy) with a median dose of 4400 cGy. The chemotherapy regimen usually consists of cyclophosphamide, doxorubicin, vincristine, and prednisone with or without rituximab.

\section{Case History}

A 62 year old female was admitted with chief complain of local non painful swelling, which increased over period of 2 months to recent size (at time of FNA $5 \times 4 \times 4 \mathrm{~cm}$ ) near sternoclavicular joint. Patient had mild fever but did not had any other B symptoms.

The patient was clinically diagnosed as case of osteosarcoma of medial head of clavicle, and patient was referred to radiology for $\mathrm{X}$ ray and Ultrasonography of local part. On X ray also suspicion of osteosarcoma of medial head of clavicle was made. And on ultrasonography lesion was described to have mild to moderate vascularity on color doppler, with few calcified areas and lytic bone destruction. On ultrasonography also, suspicion was made for malignant lesion of bone - suggestive of osteosarcoma of right clavicle. 


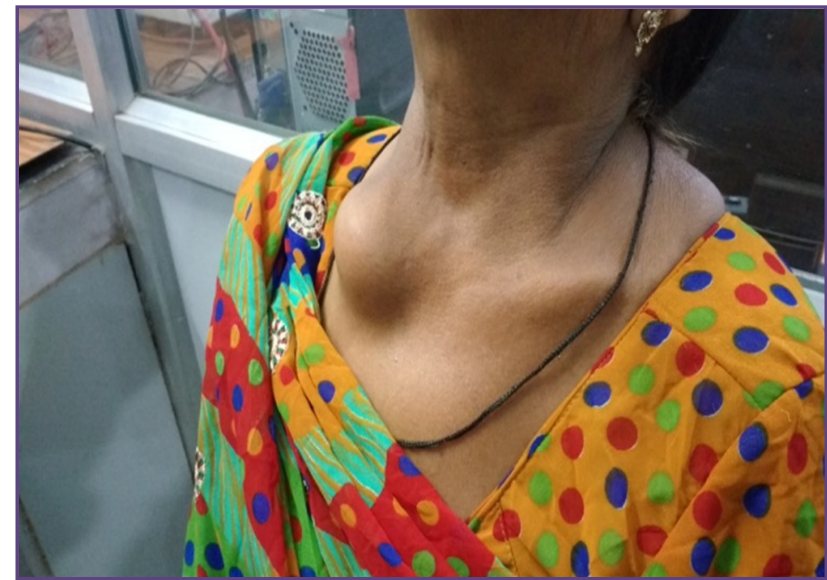

Fig. 1: Clinical image showing swelling over right sternoclavicular joint.

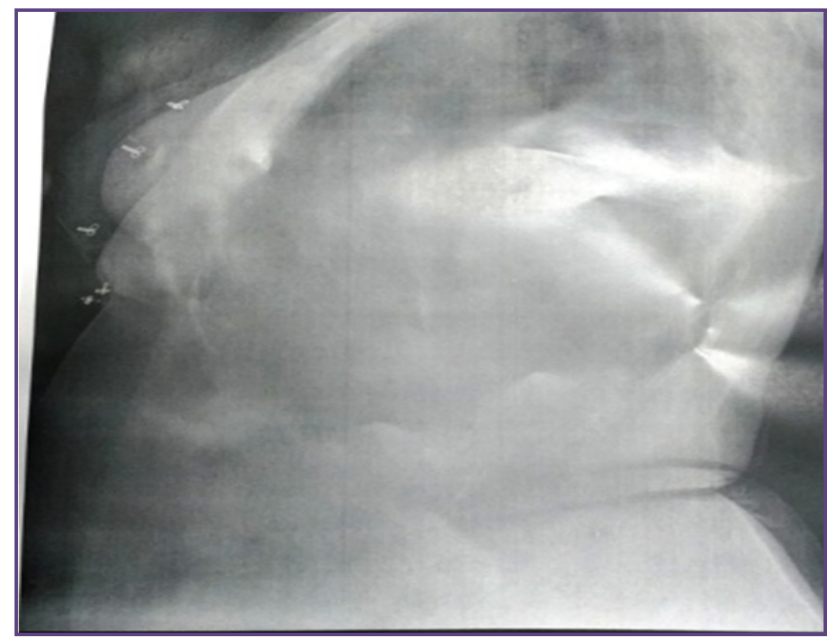

Fig3 . X-Ray lateral view showing lesion

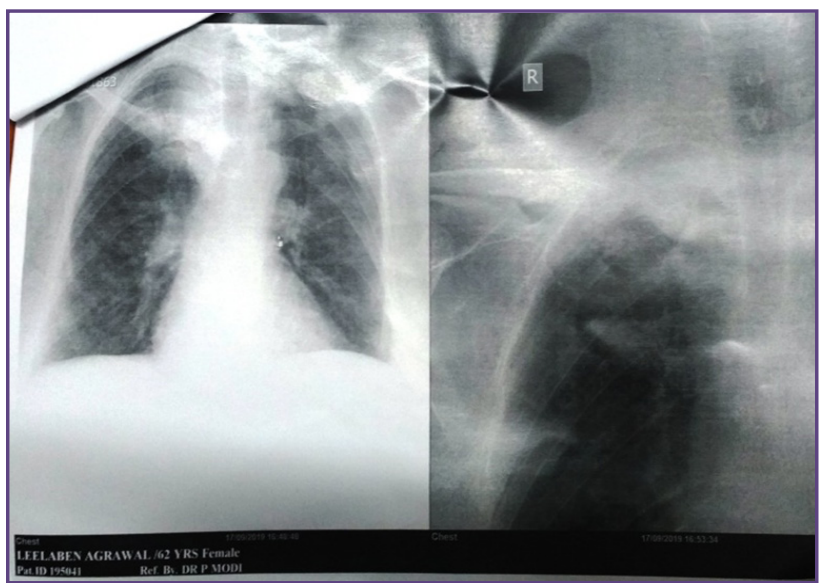

Fig. 2: X-Ray AP view showing lesion near right sterno clavicular joint.

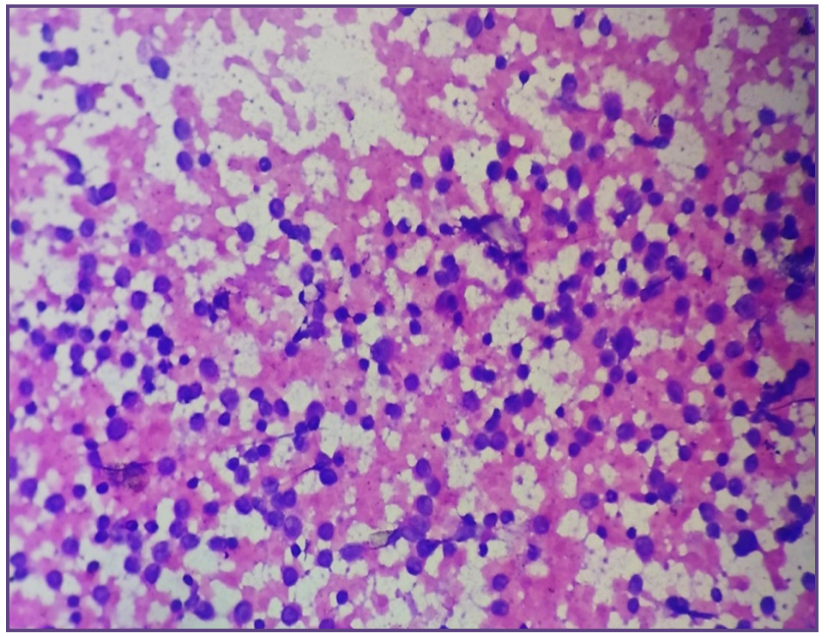

Fig. 4: Hypercellular FNA smears showing moderate to large, pleomorphic cells. (H \& E stain, 100 x Magnification).

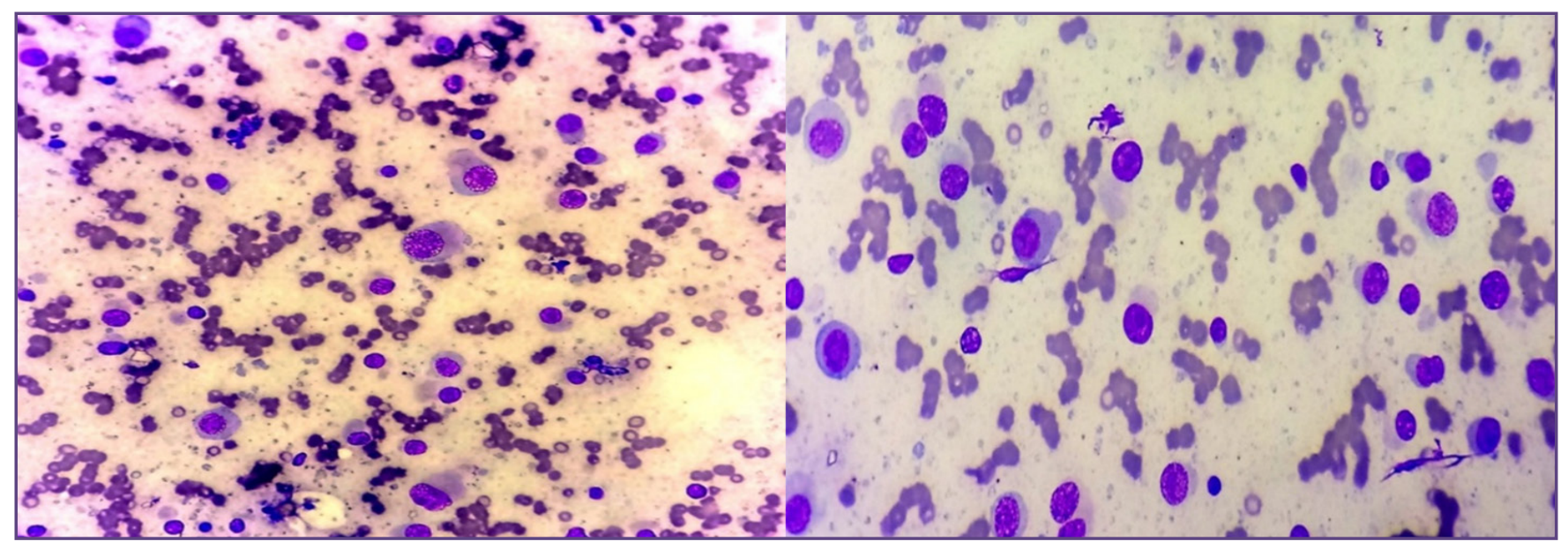

Fig. 5 - Hypercellular FNA smears showing cells which are moderate to large, pleomorphic with, pleomorphic nuclei and prominent nucleoli. Most of the cells have opened up chromatin pattern. In some of cells cytoplasm appears to be plasmacytoid. (Leishman stain, $4000 \mathrm{x}$ magnification). 


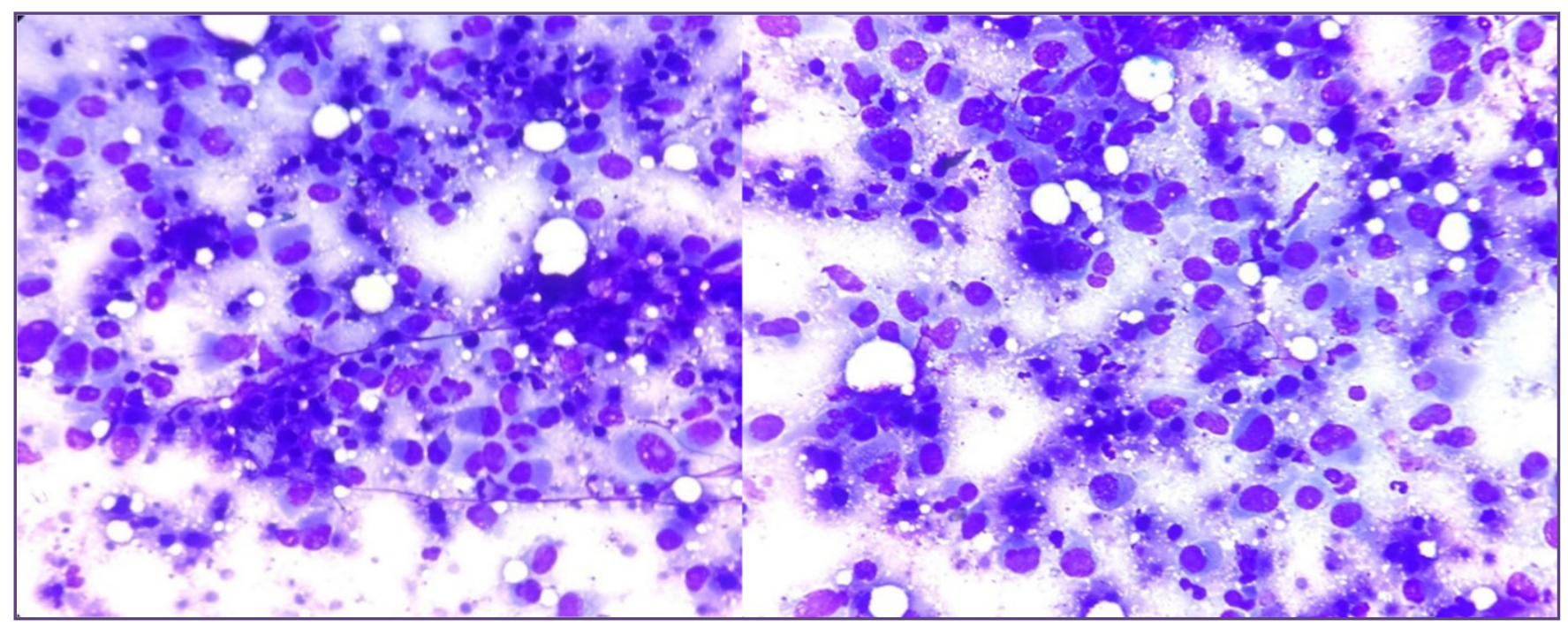

Fig. 6: Hypercellular FNA smears showing cells which are moderate to large, pleomorphic with, pleomorphic nuclei and prominent nucleoli. Most of the cells have opened up chromatin pattern. In some of cells cytoplasm appears to be plasmacytoid. (Giemsa stain, $4000 \times$ magnification).

Patient was referred to Department of Cytology for FNA investigation. FNA smears were hypercellular. Individual cells were moderate to large, pleomorphic with, pleomorphic nuclei and prominent nucleoli. Most of the cells have opened up chromatin. Also noted was evidence of bi-nucleated cells. Cytoplasm was moderate to abundant and plasmacytoid. On FNA diagnosis of lymphoproliferative disorder suggestive of non-Hodgkin lymphoma (large cell) was made. Patient was advised biopsy and IHC of lesion for confirmation of diagnosis of large cell NHL as well as subtyping of NHL.

On biopsy diagnosis of Non-Hodgkin lymphoma was confirmed (which was made on FNA), followed by application of Immunohistochemistry (IHC). On IHC tumor was having high index of Ki67 (index 85\%). Diffuse positivity was noted for MUM1, CD79a, and CD138; along with cytoplasmic positivity for ALK 1 and patchy positivity for PAX 5. Tumor was negative for BCL6, BCL2, CD20, CD5, CD23, CD10, CD19, CD4, CD8, CD7, CD3. Diagnosis of ALK positive DLBCL was given on biopsy with IHC.

Patient was referred for CT scan to check generalized lymphadenopathy and other organ involvement. On CT Scan location of lesion was unifocal, expansile lytic lesion in medial end of right clavicle causing bone erosion. No evidence of significant lymphadenopathy was noted in surrounding area.

On routine laboratory investigations, except mild anemia; patient had normal complete blood count, with normal Peripheral smear findings. Urine analysis was normal.
Patient had increased Globulin (4.60 gm/dl) and decreased albumin $(<3.60 \mathrm{gm} / \mathrm{dl})$ levels on liver function test report, with reversal of AG ratio. On electrophoresis also increase in polyclonal immunoglobulin was noted but, there was No evidence of presence of Monoclonal (paraprotein).

\section{Discussion}

Primary bone lymphoma (PBL) is rare neoplasm and there are few reported cases. Primary bone lymphoma in clavicle is rarest finding among those group. We are presenting this case as per our knowledge, Diffuse large B cell lymphoma as PBL has not been reported in literature till now in clavicle bone. Beal et al in their study of 82 patients found femur $(27 \%)$ most commonly involved followed by, pelvis $(15 \%)$, tibia/fibula $(13 \%)$, polyostotic $(13 \%)$, humerus $(12 \%)$ and spine $(9 \%)$. Cases of PBL in small bones of the hands and feet are very rare. ${ }^{[1]} \mathrm{PBL}$ can be further divided into germinal-center B-cell type and non-germinal- center B-cell type by using CD10, BCL6, and MUM1 antibodies; among which germinal-center B-cell subtype has a better prognosis. After bone biopsy confirms the diagnosis of PBL, CT Scan of the chest, abdomen, and pelvis are usually performed to exclude a primary nodal origin or distant disease. ${ }^{[2]}$ As in our case (and as per WHO definition for PBL) there should not be involvement of lymphnodes or any other organ at time of presentation.

Most common type of primary bone lymphoma is Large B cell lymphoma which usually occurs in adults. [1] Morphologically, the cells of Diffuse large B cell lymphoma are polymorphous mixture of small to large cells with multilobated nuclei, fine chromatin, and inconspicuous to prominent nucleoli. ${ }^{[2]}$ In our patient FNA smears were 
hypercellular. Individual cells were moderate to large, pleomorphic with, pleomorphic nuclei and prominent nucleoli. Most of the cells have opened up chromatin. Also noted was evidence of bi-nucleated cells. Cytoplasm was moderate to abundant and plasmacytoid. On FNA diagnosis of lymphoproliferative disorder suggestive of non-Hodgkin lymphoma (large cell) was made. Patient was advised biopsy and IHC of lesion for confirmation of diagnosis of large cell NHL as well as subtyping of NHL.

Differential diagnoses for PBL include chronic osteomyelitis, primary bone sarcoma, leukemic infiltrate, small blue round cell tumors (including Ewing sarcoma/primitive neuroectodermal tumor (PNET), rhabdomyosarcoma, metastatic neuroblastoma, and smallcell osteosarcoma), metastatic sarcomas, mesenchymal chondrosarcoma, and metastatic carcinoma. As in our case clinical as well as radiological diagnosis (with X-ray and USG of local region) was also malignant bone tumor suggestive of osteosarcoma. With use of IHC confirmation of diagnosis is made as the cells of Ewing sarcoma/PNET are monomorphic and express vimentin, CD99 (MIC2), and FLI1. They may be positive for neural markers, such as neuron-specific enolase, chromogranin, and synaptophysin. The neoplastic cells of lymphoblastic lymphoma are usually positive for terminal deoxynucleotidyl transferase (TDT). The metastatic carcinomas are positive for cytokeratin and negative for lymphoid markers. Clinical history may be helpful as well. ${ }^{[2]}$ Therefore, diagnostic samples obtained by surgical procedure and immunohistochemical tests are indispensable for clinicoradiological confirmation of PBL and for differential diagnosis. ${ }^{[4]}$ In our case also diagnosis of Non-Hodgkin lymphoma, which was made on FNA, was confirmed on biopsy, followed by application of IHC. On IHC tumor was having high index of Ki67 (index 85\%). Diffuse positivity was noted for MUM1, CD79a, and CD138; along with cytoplasmic positivity for ALK 1 and patchy positivity for PAX 5. Tumor was negative for BCL6, BCL2, CD20, CD5, CD23, CD10, CD19, CD4, CD8, CD7, CD3. Diagnosis of ALK positive DLBCL was given on biopsy with IHC. Patient was referred for CT scan to check generalized lymphadenopathy and other organ involvement. On CT Scan location of lesion was unifocal, expansile lytic lesion in medial end of right clavicle causing bone erosion. No evidence of significant lymphadenopathy was noted in surrounding area.

Ji Chen et al reported ALK positive DLBCL in nasal cavity found Immunohistology chemically ALK-positive DLBCL is characterized by the presence of ALK positive neoplastic B cells, but lack of B lymphoid lineage (CD20, CD79a), $\mathrm{T}$ lymphoid lineage (CD3, CD5) cell markers and CD30. But in our case tumor cells were positive for CD79a. The aberrant expression of ALK in these tumors exhibits a more aggressive clinical course and worse prognosis than typical diffuse large B-cell lymphoma. Anaplastic lymphoma kinase (ALK)-positive diffuse large B-cell lymphoma (DLBCL) is a rare subtype of non-Hodgkin's lymphoma (NHL) which is characterized by plasmablastic or immunoblastic morphology. This is similar case like us DLBCL positive for CD138, MUM1, ALK. ${ }^{[3]}$

\section{Conclusion}

Even though role of FNA is debatable in diagnosis of many lesions, though FNA still proves to be an important tool to suspect or diagnose, rare entities like lymphoma; at such rare clinical sites like clavicle, which may get misdiagnose clinically and radiologically. Histopathology with IHC remains confirmative tool in diagnosis and guiding further treatment.

\section{References}

1. Beal K, Allen L . Primary Bone Lymphoma: Treatment Results and Prognostic Factors with Long-Term Follow-up of 82 Patients. CANCER . 2006 ; 106(12):2652-2656.

2. Bhagavathi S . Primary Bone Lymphoma. Arch Pathol Lab Med 2009;133:1868-1871.

3. Chen J , Feng X, Dong M. Anaplastic lymphoma kinasepositive diffuse large B-cell lymphoma presenting in nasal cavity: a case report and review of literature. Int J Clin Exp Pathol 2015;8(2):2123-2130.

4. Wang J, Shouren Fan S, Jie Liu J, Bao Song B . A rare case report of primary bone lymphoma and a brief review of the literature. OncoTargets and Therapy 2016:9;4923-4928.

*Corresponding author:

Dr. Cyrus Dara Jokhi, Postal Address - 8/864, Near Tata Girls High School,Dasturwad,Navsari.Gujarat. (396445)

Phone: +91 7774884965

Email: cyrusdjokhi@gmail.com

Date of Submission : 02/06/2020

Date of Acceptance : 05/09/2020

Financial or other Competing Interests: None.

Date of Publication : 30/10/2020 